\title{
A Facile Synthesis of Cyclononatripyrroles Supplementary Information
}

\author{
Marcin Stępień† and Jonathan L. Sessler* \\ Department of Chemistry and Biochemistry, University of Texas, 1 University Station A5300, \\ Austin, TX 78712, USA
}

General Experimental ................................................. 2

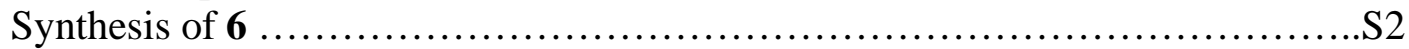

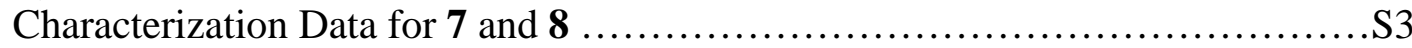

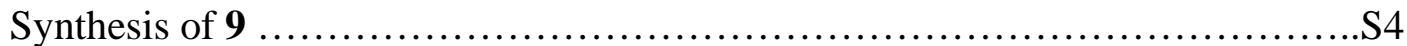

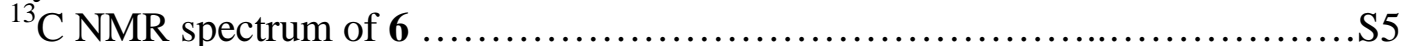

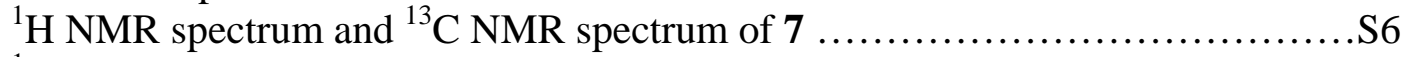

${ }^{1} \mathrm{H}$ NMR spectrum of crude 8 ............................................... 7

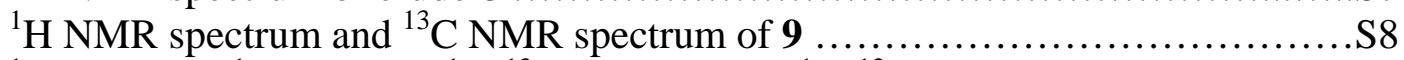

${ }^{1} \mathrm{H}$ gsCOSY, ${ }^{1} \mathrm{H}$ NOESY, ${ }^{1} \mathrm{H}_{-}{ }^{13} \mathrm{C}$ gsHSQC and ${ }^{1} \mathrm{H}_{-}{ }^{13} \mathrm{C}$ HMBC spectra of 6 .......S9-S19 
Experimental. Proton, ${ }^{13} \mathrm{C}$, and two-dimensional NMR spectra were recorded at room temperature using $300 \mathrm{MHz}$ and $500 \mathrm{MHz}$ spectrometers. Chemical shifts were referenced to residual solvent signals (7.24 ppm for $\mathrm{C}^{1} \mathrm{HCl}_{3}$ in $\mathrm{CDCl}_{3}$, $77.0 \mathrm{ppm}$ for ${ }^{13} \mathrm{CDCl}_{3}$ ). High-resolution chemical ionization (CI) mass spectra were obtained on a Waters (Micromass) Autospec mass spectrometer.

Methanol was dried by passing through two alumina columns. All other solvents and reagents were used as received. Compound 4 was obtained from (Z)-but-2-ene-1,4-diol following a literature procedure. ${ }^{1}$

Synthesis of 6. In a $500 \mathrm{~mL}$ round-bottomed flask, iodine (2.5 g) was dissolved in methanol (250 mL) with gentle heating. Compound 4 (4.76 g, $20 \mathrm{mmol})$ was added and the reaction mixture was stirred under argon for 16 hours. The mixture was then treated with excess aqueous sodium thiosulfate and extracted with dichloromethane. The combined organic extracts were dried over anhydrous sodium sulfate, filtered, and evaporated to dryness under reduced pressure. The residue was subject to flash column chromatography (silica gel, 50\% ethyl acetate in hexanes). Product $\mathbf{6}$ was collected as the first major fraction followed by smaller amounts of $\mathbf{7}$ and 8. The fractions were stripped of solvent under reduced pressure. Product 6 was recrystallized from $\mathrm{CH}_{2} \mathrm{Cl}_{2} /$ hexane. Compounds 7 and $\mathbf{8}$ were not purified further.

Triethyl 3,7,11-tris(methoxymethyl)cyclonona[1,2- $b$; 4,5- $b$ "; 7,8- $b$ "']tripyrrole-2,6,10-tricarboxylate (6): White fluffy crystals. Isolated yield: $1.3 \mathrm{~g}$ (33\%). $R_{\mathrm{f}}=0.46$ (50\% ethyl acetate/hexanes); m.p. $210{ }^{\circ} \mathrm{C} ;{ }^{1} \mathrm{H}$ NMR (500 MHz, $\left.\mathrm{CDCl}_{3}\right) 9.93$ (bs, 3H, NH), 5.04 (d, 3H, ${ }^{2} J=$ $\left.12.0 \mathrm{~Hz}, \mathrm{CH} H \mathrm{OCH}_{3}\right), 4.64$ (d, 3H, $\left.{ }^{2} J=12.0 \mathrm{~Hz}, \mathrm{CH} H \mathrm{OCH}_{3}\right), 4.17-4.29\left(\mathrm{~m}, 6 \mathrm{H}, \mathrm{OCH}_{2} \mathrm{CH}_{3}\right)$, 4.05 (d, 3H, ${ }^{2} J=14.9 \mathrm{~Hz}$, bridge $\mathrm{CH} H$ ), 3.61 (d, 3H, ${ }^{2} J=14.9 \mathrm{~Hz}$, bridge $\mathrm{CH} H$ ), 3.40 (s, 9H, $\left.\mathrm{CH}_{2} \mathrm{OCH}_{3}\right), 1.31\left(\mathrm{t}, 9 \mathrm{H},{ }^{3} \mathrm{~J}=7.2 \mathrm{~Hz}, \mathrm{OCH}_{2} \mathrm{CH}_{3}\right) ;{ }^{13} \mathrm{C} \mathrm{NMR}\left(126 \mathrm{MHz}, \mathrm{CDCl}_{3}\right) \delta 160.82$ 
( $\left.{ }^{13} \mathrm{COOEt}\right), 133.32$ (pyrrole 2-C), 124.47 (pyrrole 4-C), 119.21 (pyrrole 3-C), 117.66 (pyrrole 5C), $64.91\left({ }^{13} \mathrm{CH}_{2} \mathrm{OCH}_{3}\right), 59.80\left(\mathrm{O}^{13} \mathrm{CH}_{2} \mathrm{CH}_{3}\right), 57.29\left(\mathrm{CH}_{2} \mathrm{O}^{13} \mathrm{CH}_{3}\right)$, 20.98 (bridge $\left.{ }^{13} \mathrm{CH}_{2}\right), 14.52$ $\left(\mathrm{OCH}_{2}{ }^{13} \mathrm{CH}_{3}\right)$; HR-MS (CI+): $m / z$ 585.2687 [M+], calcd for $\mathrm{C}_{30} \mathrm{H}_{39} \mathrm{~N}_{3} \mathrm{O}_{9}$ : 585.2686. Elemental analysis for $\mathrm{C}_{30} \mathrm{H}_{39} \mathrm{~N}_{3} \mathrm{O}_{9}$ : calcd. C 61.53, H 6.71, N 7.18; found C 61.78, H 6.65, N 7.13.

Ethyl 5-((5-(ethoxycarbonyl)-4-(methoxymethyl)-1H-pyrrol-3-yl)methyl)-3,4-bis(methoxymethyl)-pyrrole-2-carboxylate (8) Viscous yellow oil. Yield: ca. $0.6 \mathrm{~g}$ (crude). $R_{\mathrm{f}}=0.23$ (50\% ethyl acetate/hexanes); ${ }^{1} \mathrm{H}$ NMR (300 MHz, $\left.\mathrm{CDCl}_{3}\right) \delta 10.41$ (bs, 1H, NH), 9.02 (bs, 1H, NH), $6.71\left(\mathrm{~d}, 1 \mathrm{H},{ }^{4} \mathrm{~J}=3.0 \mathrm{~Hz}, \alpha-\mathrm{H}\right), 4.80\left(\mathrm{~s}, 2 \mathrm{H}, \mathrm{CH}_{2} \mathrm{OCH}_{3}\right), 4.66$ (s, $\left.2 \mathrm{H}, \mathrm{CH}_{2} \mathrm{OCH}_{3}\right), 4.44$ (s, $2 \mathrm{H}$, $\mathrm{CH}_{2} \mathrm{OCH}_{3}$ ), 4.31 (q, $2 \mathrm{H},{ }^{4} J=7.3 \mathrm{~Hz}, \mathrm{CH}_{2} \mathrm{CH}_{3}$ ), 4.25 (q, $2 \mathrm{H},{ }^{4} J=7.3 \mathrm{~Hz}, \mathrm{CH}_{2} \mathrm{CH}_{3}$ ), 3.50 (s, 3H, $\mathrm{CH}_{2} \mathrm{OCH}_{3}$ ), 3.34 (s, $3 \mathrm{H}, \mathrm{CH}_{2} \mathrm{OCH}$ ), 3.33 (s, 3H, $\mathrm{CH}_{2} \mathrm{OCH}_{3}$ ), 1.35 (t, $3 \mathrm{H},{ }^{4} \mathrm{~J}=7.3 \mathrm{~Hz}, \mathrm{CH}_{2} \mathrm{CH}_{3}$ ), $1.30\left(\mathrm{t}, 3 \mathrm{H},{ }^{4} \mathrm{~J}=7.3 \mathrm{~Hz}, \mathrm{CH}_{2} \mathrm{CH}_{3}\right)$.

Ethyl 3,4-bis(methoxymethyl)pyrrole-2-carboxylate (7). Viscous yellow oil. Yield: $0.59 \mathrm{~g}$ (13\%). $R_{\mathrm{f}}=0.30$ (50\% ethyl acetate/hexanes); ${ }^{1} \mathrm{H}$ NMR (300 MHz, $\left.\mathrm{CDCl}_{3}\right) \delta 9.14$ (bs, $\left.1 \mathrm{H}, \mathrm{NH}\right)$, $6.87\left(\mathrm{~d}, 1 \mathrm{H},{ }^{4} J=3.0 \mathrm{~Hz}, \alpha-\mathrm{H}\right), 4.68$ (s, $2 \mathrm{H}, \mathrm{CH}_{2} \mathrm{OCH}_{3}$ ), 4.39 (s, $2 \mathrm{H}, \mathrm{CH}_{2} \mathrm{OCH}_{3}$ ), 4.30 (q, $2 \mathrm{H},{ }^{4} J$ $=7.3 \mathrm{~Hz}, \mathrm{CH}_{2} \mathrm{CH}_{3}$ ), 3.341 (s, 3H, $\left.\mathrm{CH}_{2} \mathrm{OCH}_{3}\right), 3.337$ (s, 3H, $\left.\mathrm{CH}_{2} \mathrm{OCH}_{3}\right), 1.34$ (t, 3H, ${ }^{4} J=7.3 \mathrm{~Hz}$, $\left.\mathrm{CH}_{2} \mathrm{CH}_{3}\right) .{ }^{13} \mathrm{C}$ NMR $\left(75 \mathrm{MHz}, \mathrm{CDCl}_{3}\right) \delta 161.11,125.49,123.04,121.44,120.87,66.22,64.45$, $60.35,57.83,57.82,14.39$. 
Synthesis of 9. In a $25 \mathrm{~mL}$ round bottomed flask equipped with a reflux condenser and a magnetic stirring bar were placed 6 (50 mg, $85.5 \mu \mathrm{mol}$ ), benzyl alcohol (89 $\mu \mathrm{L} ; 855 \mu \mathrm{mol}$ ), and toluene $(8 \mathrm{~mL}) \cdot p$-Toluenesulfonic acid $(1 \mathrm{mg})$ was then added and the mixture was stirred and heated to reflux under argon and maintained that way for 20 hours. Subsequently, the reaction was allowed to cool to room temperature and the solvent was removed under reduced pressure. The crude product was purified by recrystallization from $\mathrm{CH}_{2} \mathrm{Cl}_{2} /$ methanol and $\mathrm{CH}_{2} \mathrm{Cl}_{2} /$ hexane. Yield $54 \mathrm{mg}$ (78\%). $R_{\mathrm{f}}=0.57$ (50\% AcOEt / hexanes); m.p. $145{ }^{\circ} \mathrm{C} ;{ }^{1} \mathrm{H}$ NMR $(300 \mathrm{MHz}$, $\left.\mathrm{CDCl}_{3}\right) \delta 10.00$ (bs, 3H, NH), 7.43 (m, 6H, $o-\mathrm{Ph}$ ), 7.26-7.37 (m, 9H, $\left.m, p-\mathrm{Ph}\right), 5.10$ (d, 3H, ${ }^{2} J=$ $12.1 \mathrm{~Hz}, \mathrm{CH} H \mathrm{OCH} \mathrm{H}_{2} \mathrm{Ph}$ ), 4.74 (d, 3H, ${ }^{2} J=12.9 \mathrm{~Hz}, \mathrm{CH}_{2} \mathrm{OCH} H \mathrm{Ph}$ ), 4.62 (d, 3H, ${ }^{2} J=12.1 \mathrm{~Hz}$, $\mathrm{CHHOCH}_{2} \mathrm{Ph}$ ), 4.59 (d, 3H, ${ }^{2} J=12.9 \mathrm{~Hz}, \mathrm{CH}_{2} \mathrm{OCH} H \mathrm{Ph}$ ), 4.052 (d, 3H, ${ }^{2} J=15.0 \mathrm{~Hz}$, bridge

$\mathrm{CH} H$ ), $4.047\left(\sim \mathrm{q}, 6 \mathrm{H},{ }^{3} J=7.3 \mathrm{~Hz}, \mathrm{OCH}_{2} \mathrm{CH}_{3}\right), 3.66$ (d, 3H, ${ }^{2} J=15.0 \mathrm{~Hz}$, bridge $\mathrm{CH} H$ ), 1.06 (t, $\left.9 \mathrm{H},{ }^{3} J=7.3 \mathrm{~Hz}, \mathrm{OCH}_{2} \mathrm{CH}_{3}\right) ;{ }^{13} \mathrm{C}$ NMR $\left(75 \mathrm{MHz}, \mathrm{CDCl}_{3}\right) \delta 160.81,138.17,133.57,128.41$, 128.13, 127.65, 124.56, 119.06, 117.50, 71.63, 62.43, 59.78, 21.05, 14.28; HR-MS (CI+): m/z 813.3622 [M+ $]$, calcd for $\mathrm{C}_{48} \mathrm{H}_{51} \mathrm{~N}_{3} \mathrm{O}_{9}: 813.3625$.

\section{References for Supporting Information}

1. Johnstone, K. D.; Pearce, W. E.; Pyke, S. M. J. Porphyrins Phthalocyanines 2002, 6, 661. 

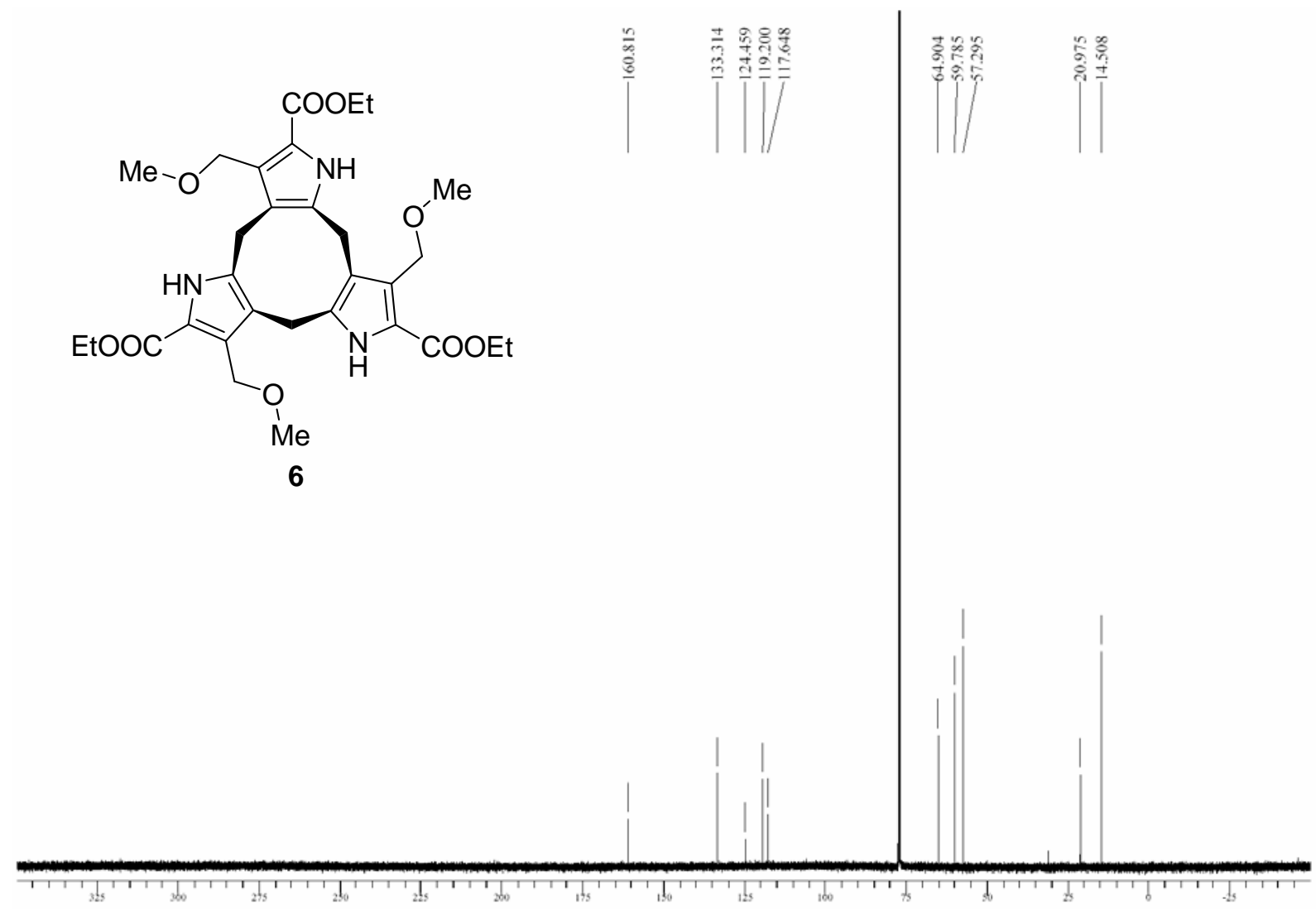

Figure S1. ${ }^{13} \mathrm{C}$ NMR spectrum $(126 \mathrm{MHz})$ of 6 . 

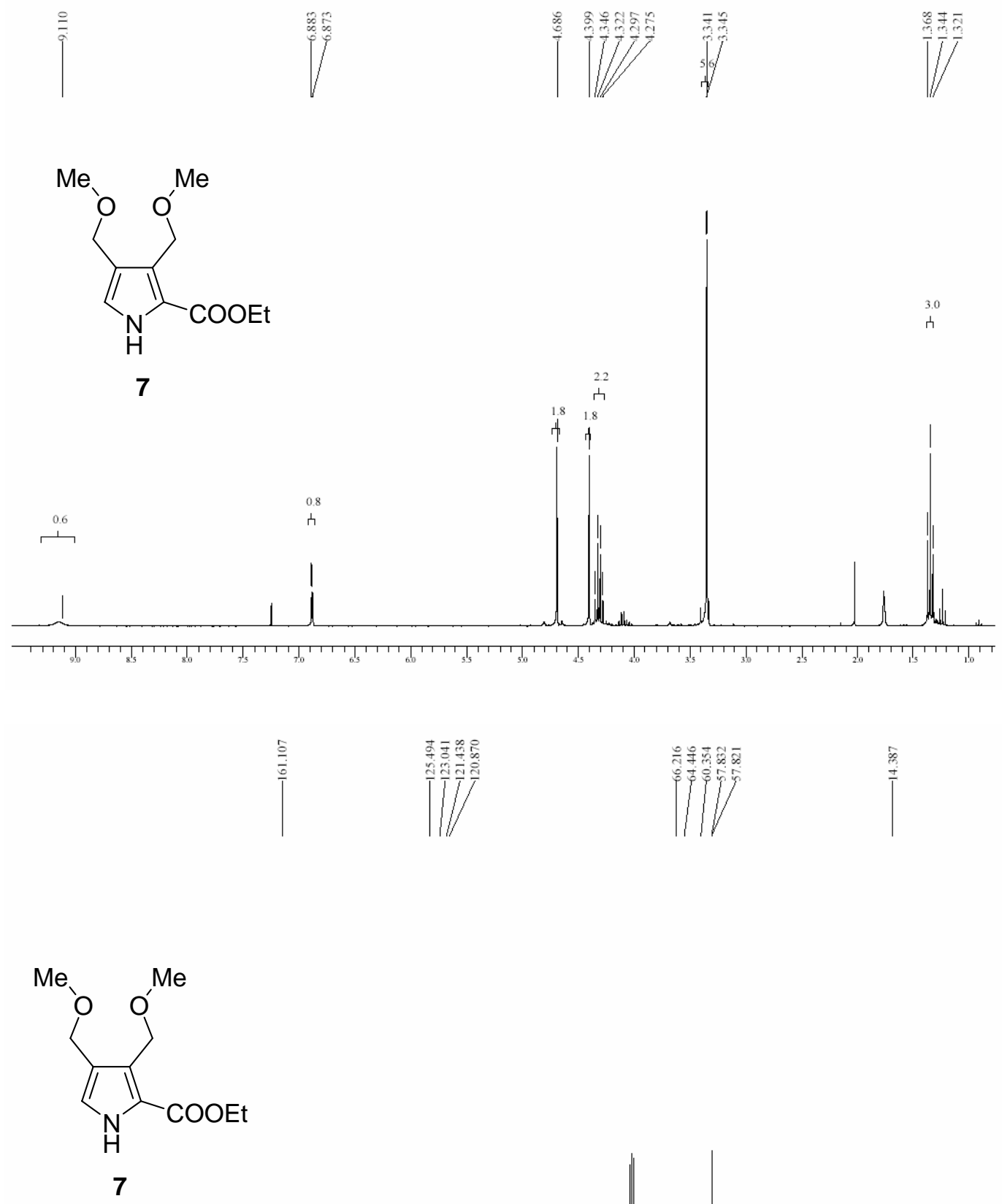


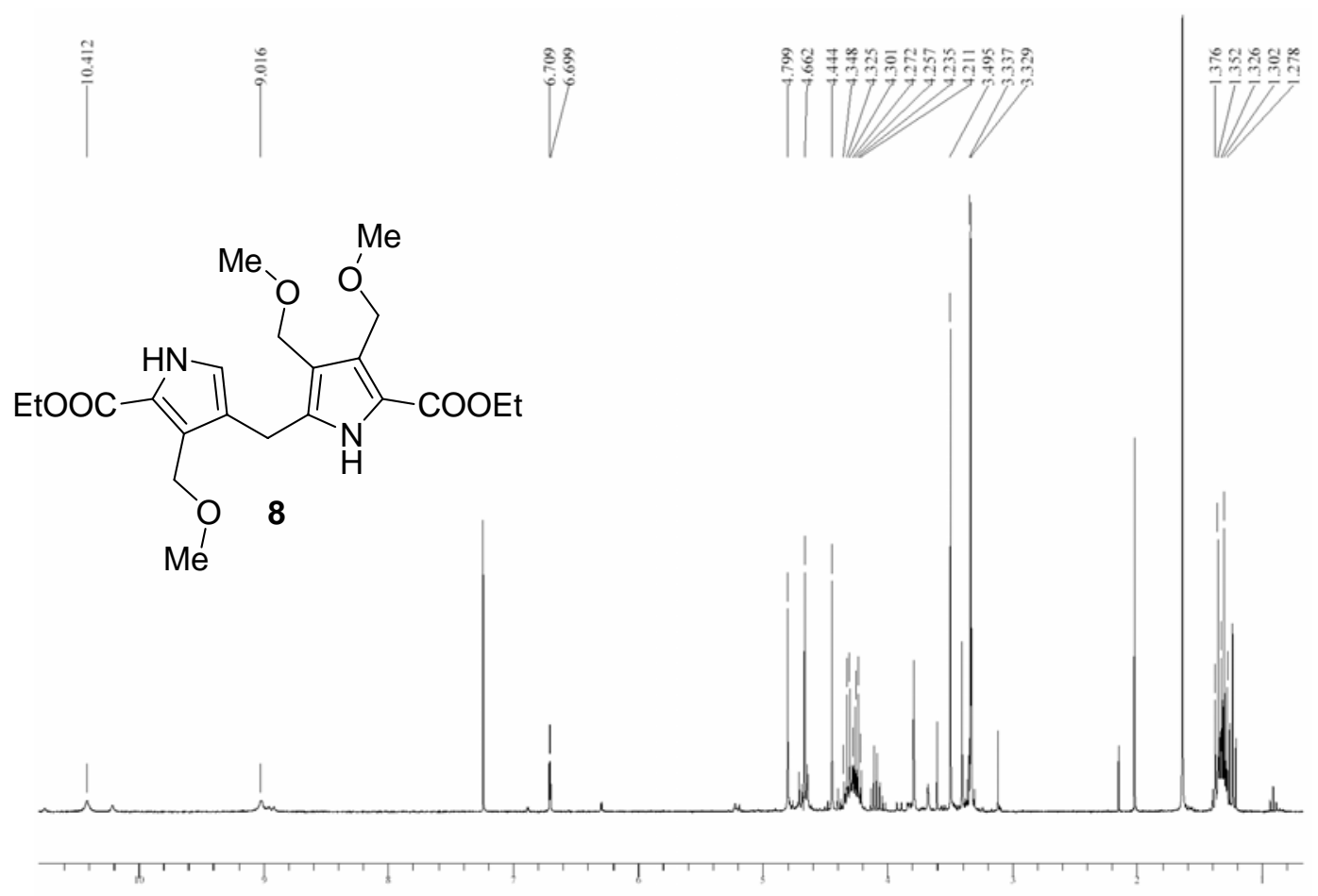

Figure S3. ${ }^{1} \mathrm{H}$ NMR spectrum (300 MHz) of crude 8. 

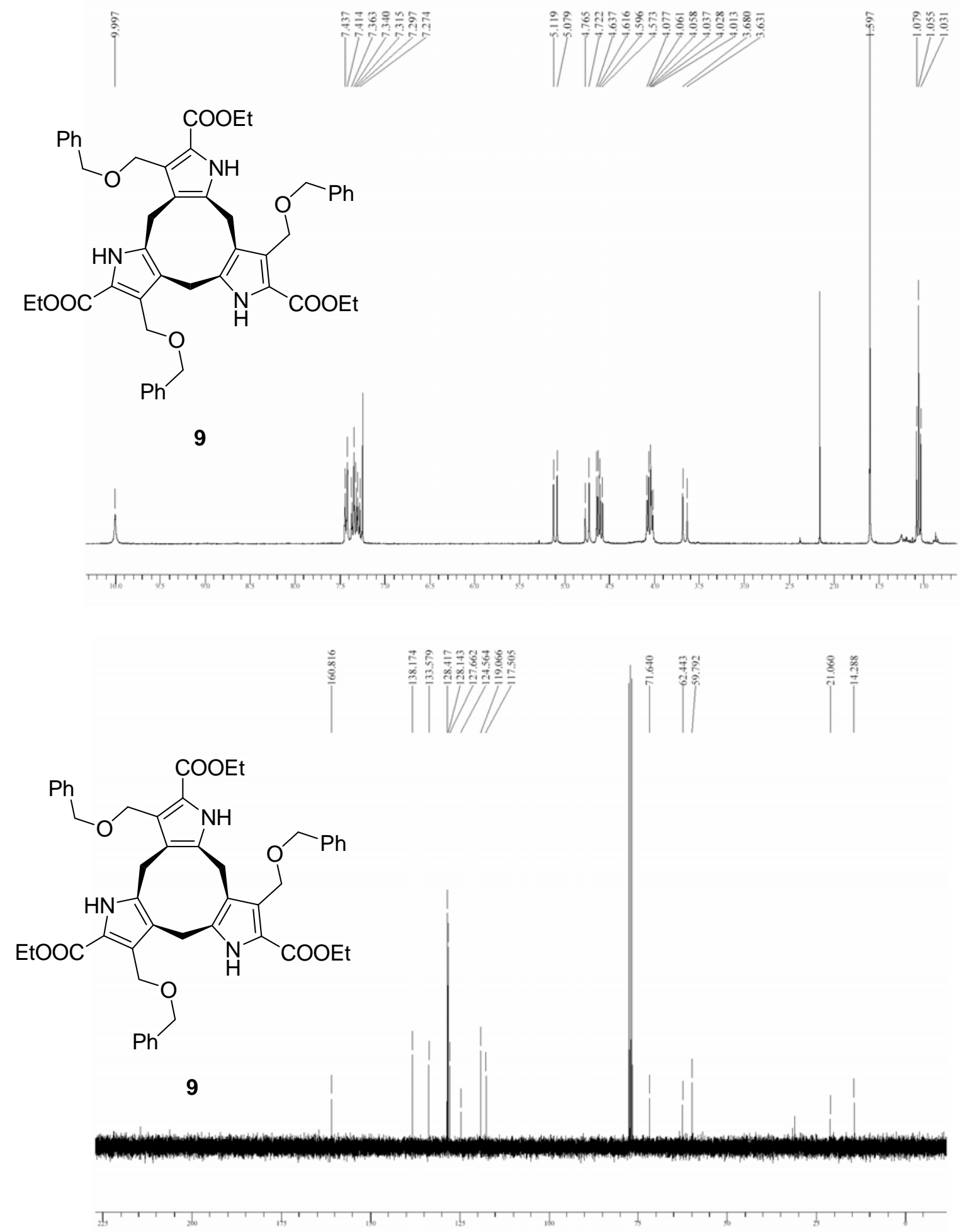

Figure S4. ${ }^{1} \mathrm{H}$ NMR spectrum (300 MHz, top) and ${ }^{13} \mathrm{C}$ NMR spectrum (75 MHz, bottom) of 9. 
Figures S5-S15. ${ }^{1} \mathrm{H}$ gsCOSY, ${ }^{1} \mathrm{H}$ NOESY, ${ }^{1} \mathrm{H}^{-13} \mathrm{C}$ gsHSQC and ${ }^{1} \mathrm{H}-{ }^{13} \mathrm{C}$ HMBC spectra of 6 .

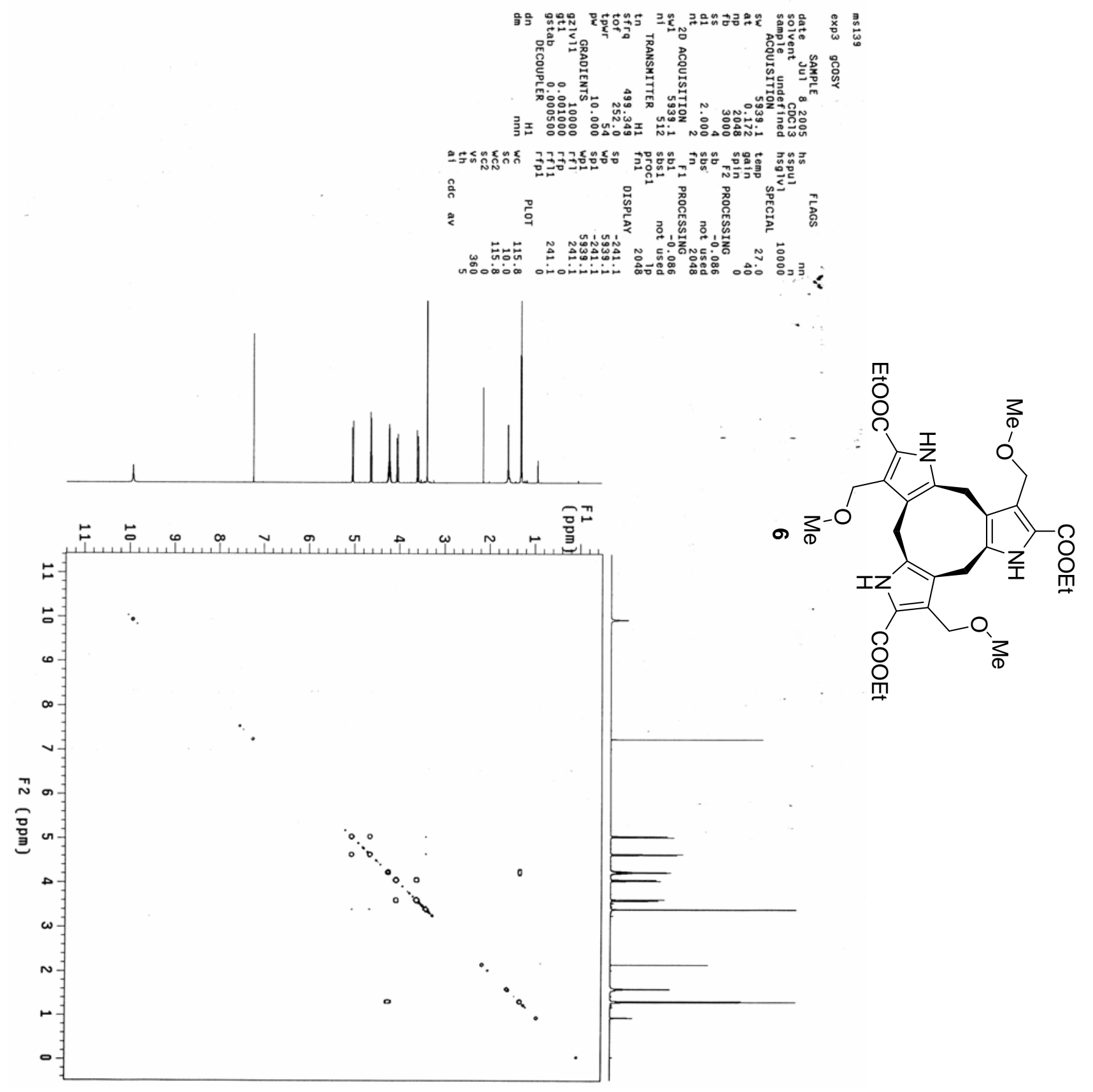




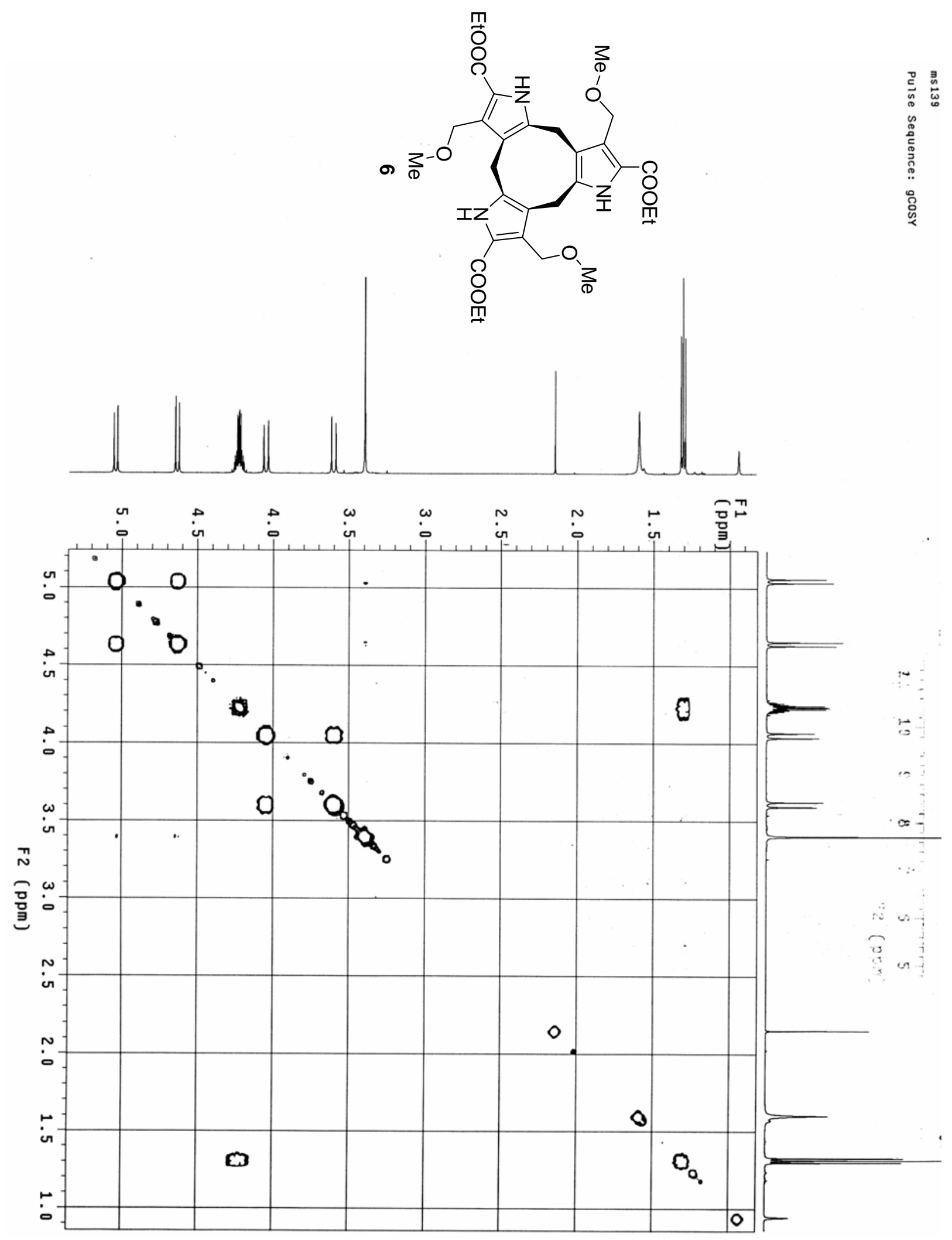




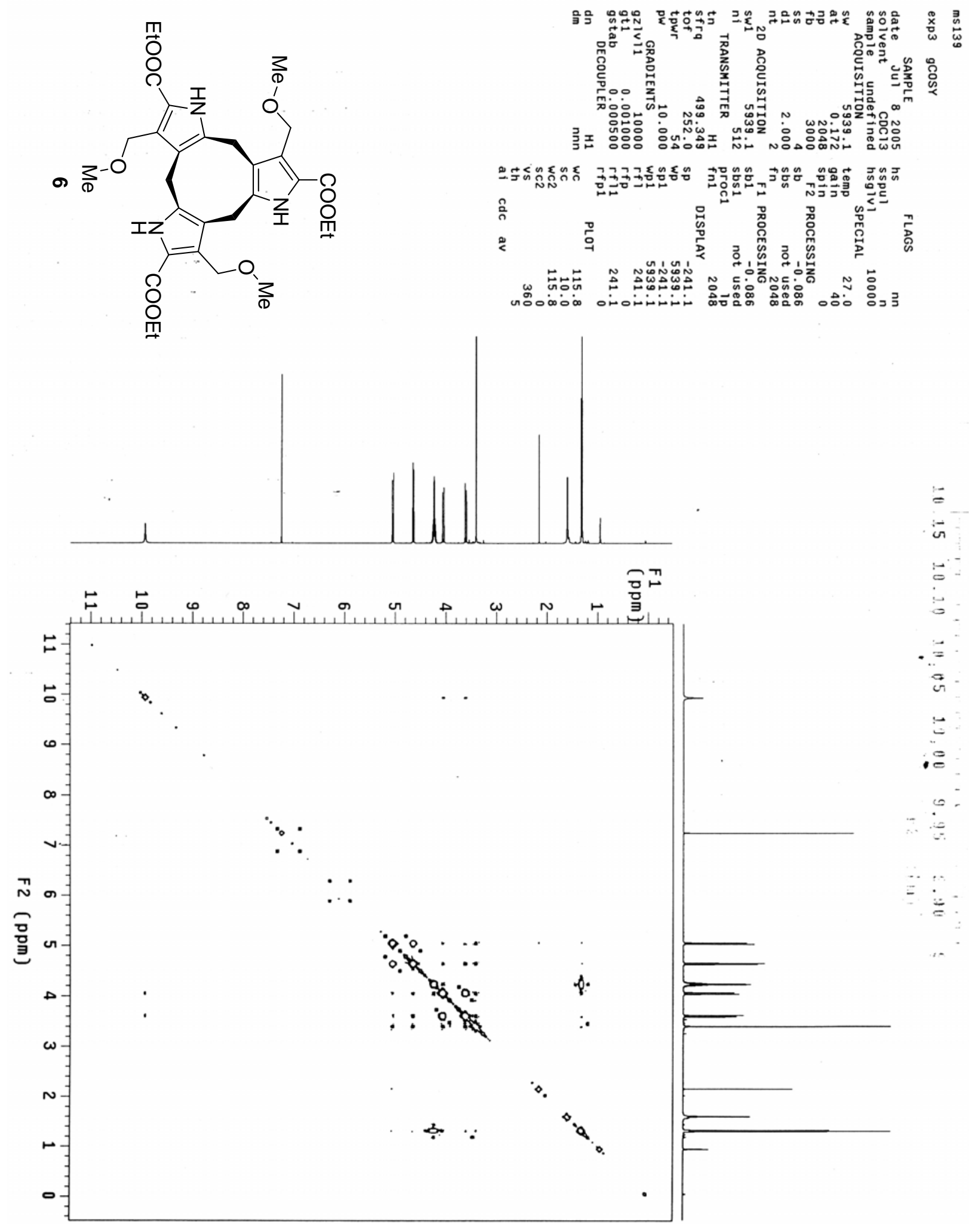




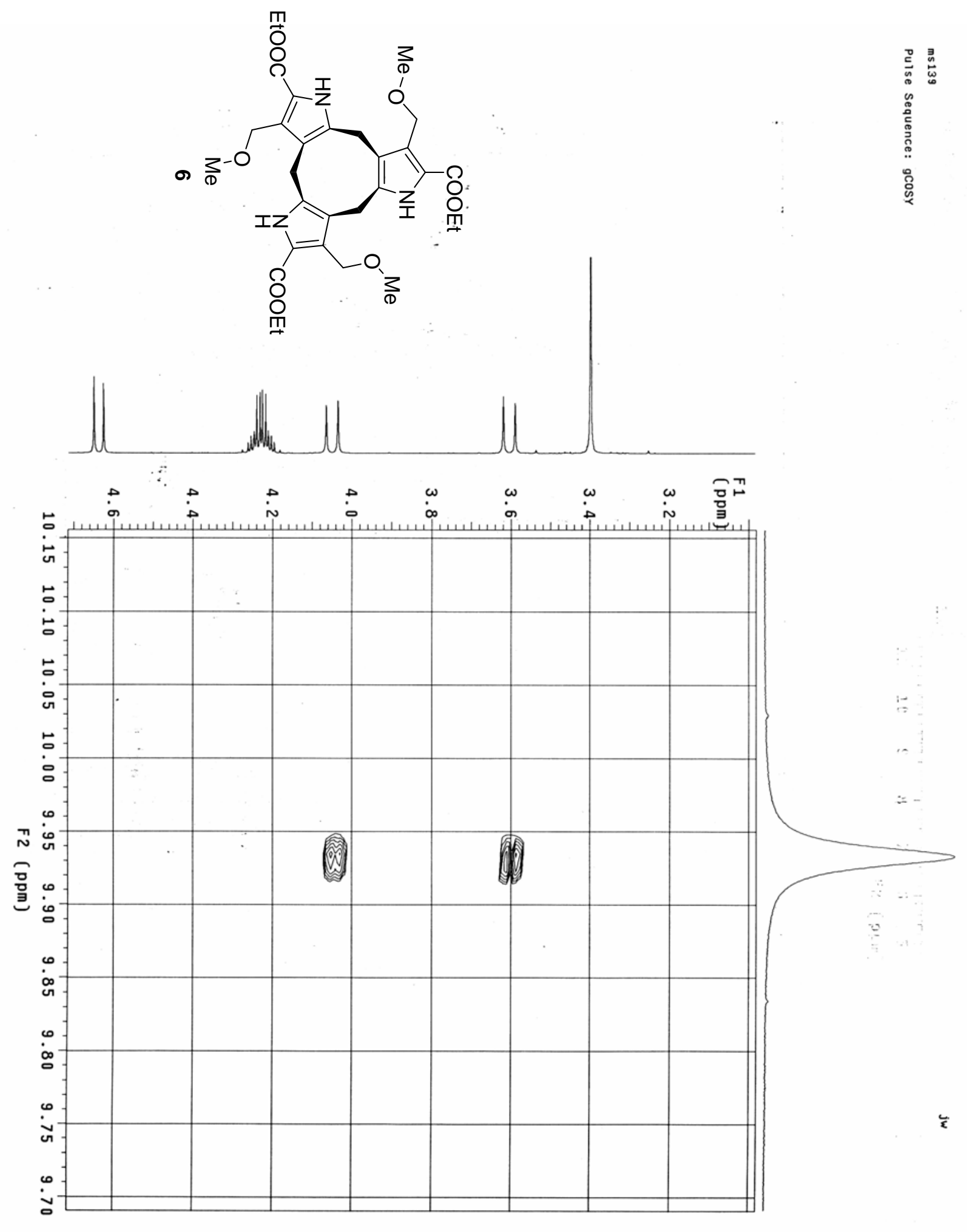



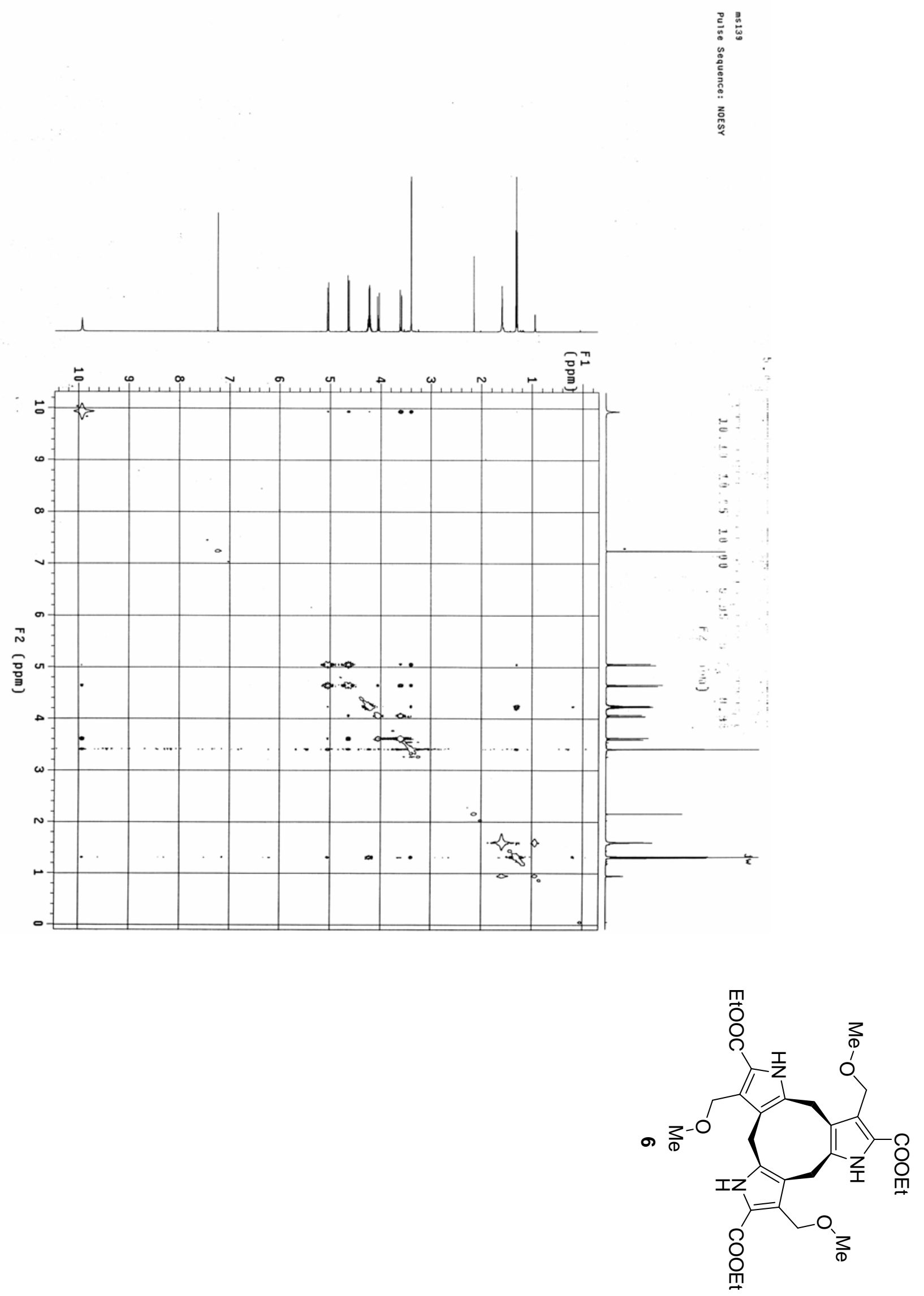


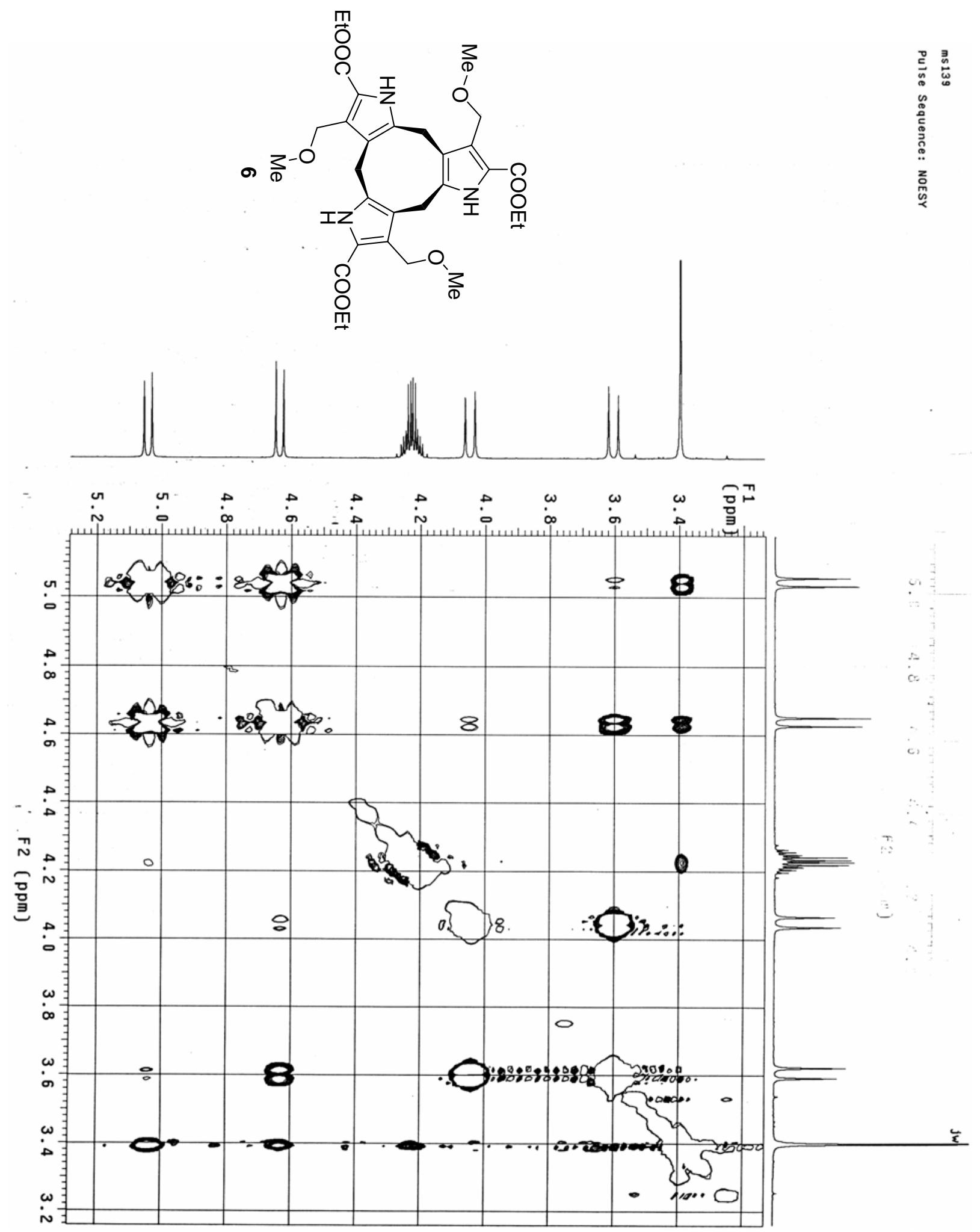




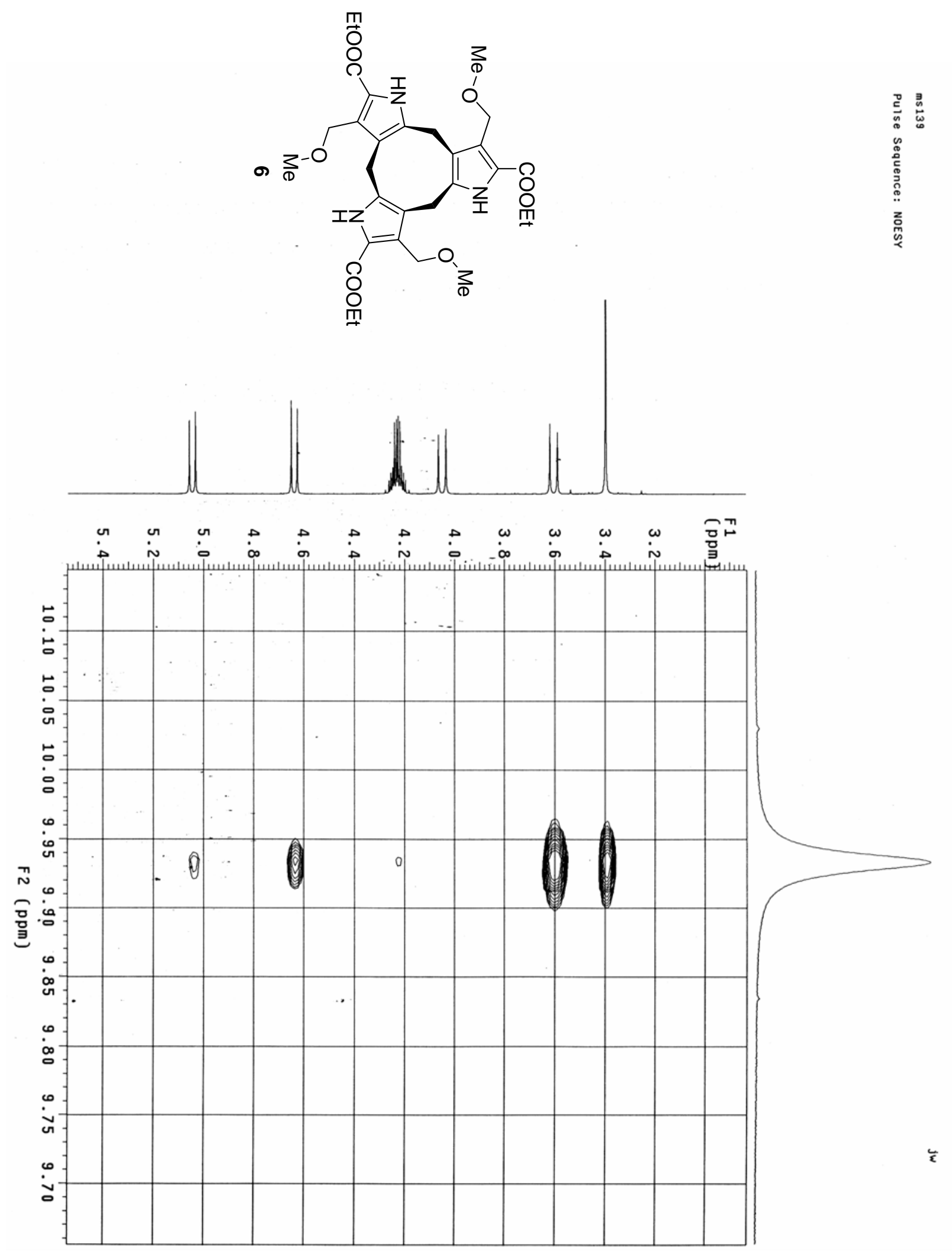



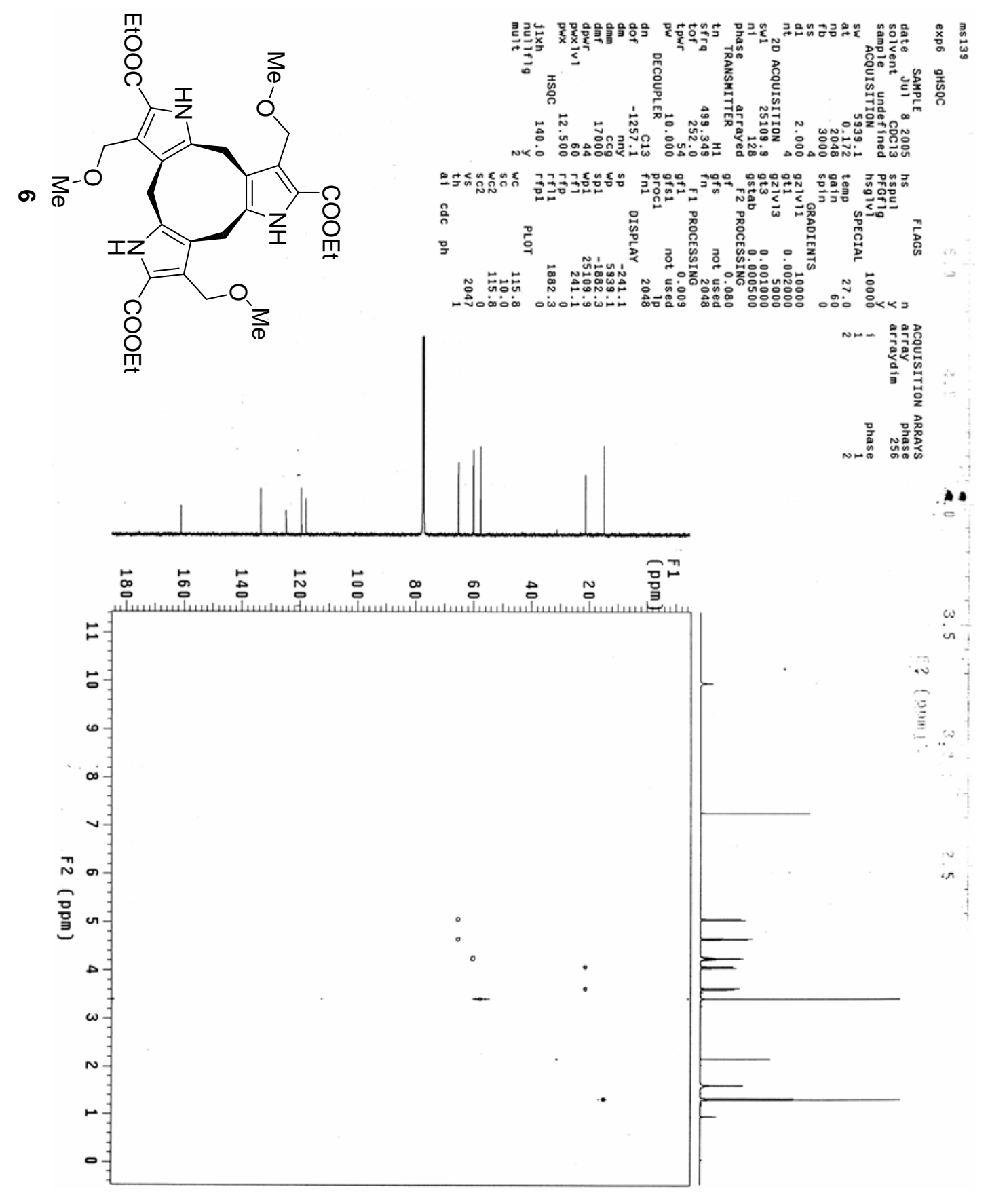


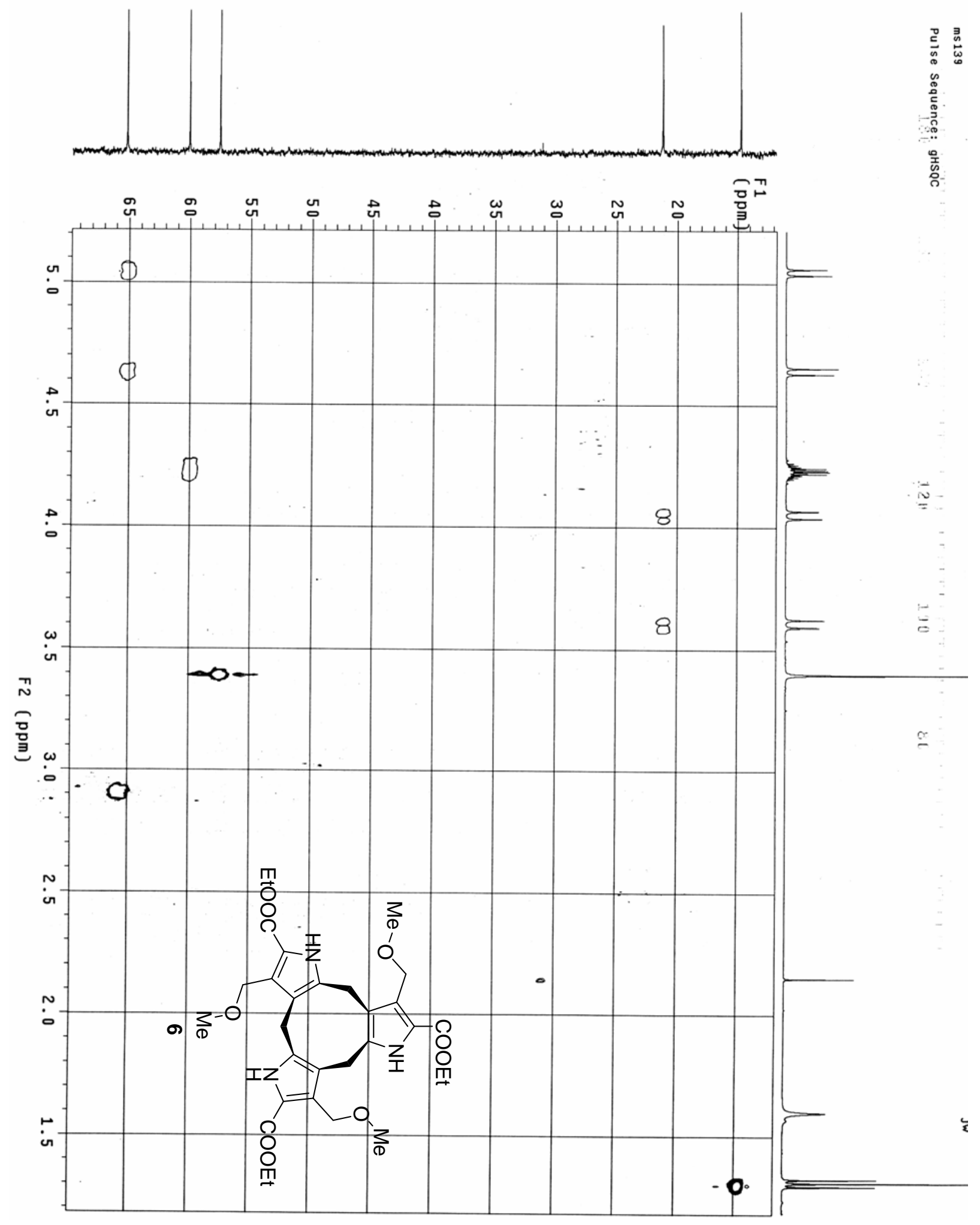



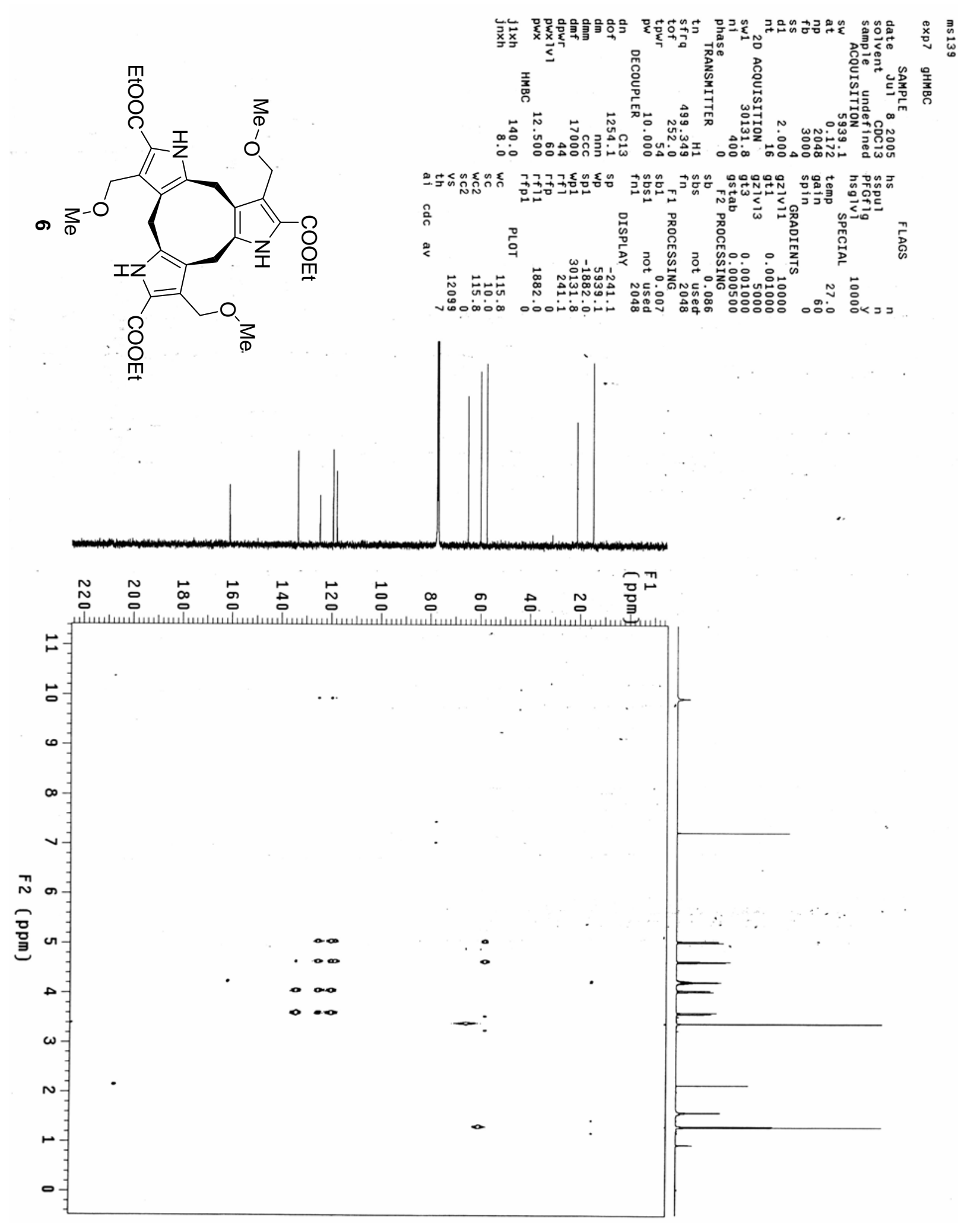


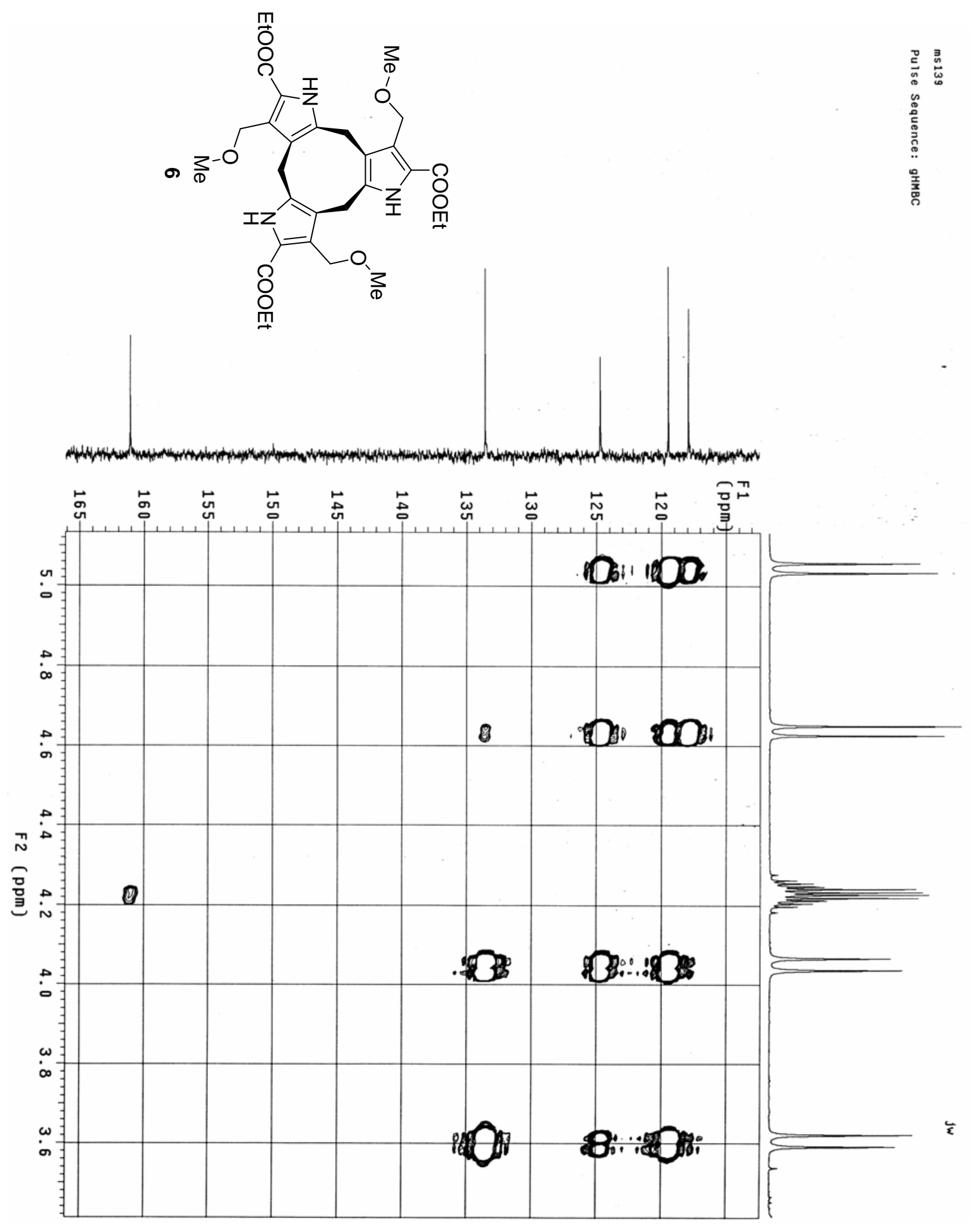

